\title{
Aprendiendo con las abejas en el primer ciclo de Educación Infantil
}

\author{
María del Mar Prieto López y Antonio Torralba-Burrial 23 \\ ${ }^{1}$ Escuela de Educación Infantil El Franco. La Caridad. España; ${ }^{2}$ Dpto. Ciencias de la \\ Educación. Universidad de Oviedo. Oviedo. España; ${ }^{3}$ Instituto de Recursos Naturales \\ y Ordenación del Territorio (Indurot). Universidad de Oviedo. Mieres. España
}

[Recibido el 3 de mayo de 2021, aceptado el 05 de septiembre de 2021]

Existe una desconexión creciente entre la sociedad en general, y en la infancia en particular, con la naturaleza y el medio en el que vivimos, por lo que se ha desarrollado esta experiencia didáctica buscando una conexión en la primera infancia facilitada desde la Educación Ambiental en la escuela. Se ha diseñado esta secuencia didáctica basada en las abejas, insectos polinizadores empleados como recurso didáctico interrelacionando el entorno natural y cultural, implementándola en el primer ciclo de Educación Infantil en una escuela rural de Asturias. Se han preparado recursos didácticos y actividades adaptadas, combinando el aula, el patio escolar, el huerto escolar y el aprendizaje más allá del aula, abogando por la importancia de la biodiversidad, del aprovechamiento diversificado de los recursos naturales y de la conexión y relación directa con la naturaleza desde edades tempranas, resultando bien acogida por el alumnado, especialmente las actividades manipulativas y al aire libre.

Palabras clave: Educación Infantil, Insectos, apicultura; recursos naturales; Educación Ambiental.

\section{Learning with bees during the first stage of Early Childhood Education}

In view of the growing disconnect between society in general and childhood in particular and nature and the environment in which we live, a didactic experience was created to re-establish that connection from early childhood through Environmental Education at school. A teaching sequence was designed based on bees, a pollinating insect used as a teaching resource to interrelate the natural and cultural environments, and implemented among children aged $0-3$ years at a rural school in Asturias. Teaching resources and adapted activities were created, combining the classroom, the schoolyard, the school garden and learning beyond the classroom, to highlight the importance of biodiversity, diversified use of natural resources and establishing a direct relationship with the natural environment from an early age. The experience was very well received by the children, especially the hands-on and outdoor activities.

Keywords: Early Childhood Education; insects; beekeeping; natural resources; Environmental Education.

Para citar el artículo. Prieto López, M. M. y Torralba-Burrial, A. (2021). Aprendiendo con las abejas en el primer ciclo de Educación Infantil. Ápice. Revista de Educación Científica, 5(2), 55-70. DOI: https://doi.org/10.17979/arec.2021.5.2.8262

Contacto.mprietolopez.11@gmail.com0 torralbaantonio@uniovi.es 


\section{Introducción}

Nos encontramos inmersos en un momento de crisis ecológica y problemas ambientales, con tendencias alarmantes para el medioambiente global. Ante ello, se plantea la necesidad de una transición ecológica que facilite la recuperación medioambiental, la biodiversidad y la consecución de los Objetivos de Desarrollo Sostenible (ODS) impulsados por Naciones Unidas. Aunque se incrementa esta sensación general de necesidad de conservar el medioambiente, lo cierto es que existen patrones de desconexión creciente entre la sociedad y la naturaleza (Kesebir y Kesebir, 2017), que dificultan la construcción de conocimientos sólidos sobre el medioambiente cercano (Almeida, García Fernández y Strecht-Ribeiro, 2020). Para mitigarlo resulta necesario reconectar a la sociedad con la naturaleza (Pyle, 2003) favoreciendo las experiencias con la biodiversidad y el medioambiente desde la infancia (Freire, 2011; Louv, 2016), que familiaricen al alumnado con el gusto por la naturaleza y con las que puedan construir conocimientos que potencien una ciudadanía futura comprometida con la sostenibilidad (Lumber, Richardson y Sheffield, 2017; Ives et al., 2018).

Si bien los planteamientos relacionados con una educación más cercana a la naturaleza no son recientes (Fröbel y los Kindergarten, Rosa Sensat y la Escola del Bosc serían referencias clásicas, véase en contexto Colmenar, 1989; Sensat, 1933), sí que es cierto que en los últimos años estilos relacionados con las Escuelas del Bosque y en la naturaleza están incrementándose (García-González y Schenetti, 2019). Autores como Louv (2008) plantean que la desconexión de la naturaleza en la infancia puede generar problemas de "déficit de naturaleza", resultando necesario revertir la desconexión con la misma. En efecto, se ha comprobado el efecto amortiguador de la naturaleza sobre el estrés de los niños (Corraliza y Collado, 2011).

En este sentido, el primer ciclo de educación infantil, aunque muchas veces ausente en las publicaciones de didáctica de la ciencia, sí que permite trabajar el aprendizaje desde la observación, manipulación y experimentación científicas (Vega, 2006), siendo recomendable plantear experiencias en el medio natural desde la primera infancia (Ardoin y Bowers, 2020; Torres-Porras, Alcántara, Arrebola, Rubio y Mora, 2017).

Teniendo esto en cuenta, se ha planteado este trabajo como proceso de enseñanza-aprendizaje de la biodiversidad a partir de unos insectos concretos en los que se imbrican el medio natural con su vertiente cultural de una forma muy clara: las abejas. En efecto, las experiencias cercanas con estos insectos pueden facilitar que el alumnado integre su entorno natural con el cultural, al tiempo que toma consciencia de sus propias relaciones desde dentro de ese entorno, y no desde una perspectiva separada (Atkinson, 2015). Así, se pretende acercar las abejas y la apicultura al alumnado, a través de experiencias observacionales, manipulativas y sensoriales, buscando el desarrollo global del alumnado y el contacto con el medio ambiente.

Las experiencias educativas con las abejas en sentido amplio, y no solo la abeja melífera doméstica, pueden contribuir a una mayor sensibilidad ambiental y relaciones más empáticas con los animales (Atkinson, 2015; Nxumalo, 2017). Las propuestas para trabajar las abejas en la escuela son variadas, pero generalmente centradas en la Educación Primaria (Fisher Miller y Jamison, 2014; Hart, 1987; García, Rosado y Hernaz, 2012), siendo muy escasas las aproximaciones que incluyan Educación Infantil (Echols, 1999; Montero, Conde y Sánchez, 2016; Rodríguez-Loinaz, Toral y Palacios-Agundez, 2018; Valin, Moledo, López Maceiras y García-Rodeja, 2012), sin referencias a proyectos educativos estructurados en el Primer Ciclo de Educación Infantil. No obstante, la observación y la exploración, el acercamiento y vinculación con la naturaleza, la observación de algunas características de los 
seres vivos o la curiosidad hacia ellos están recogidas en el currículo del ciclo (Decreto 113/2014). Y aunque no son frecuentes, observaciones y actividades con otros invertebrados en la etapa 0-3 (Inoue, 2018), en la clase de 3 años (Cutter-Mackenzie, Edwards, Moore y Boyd, 2014) o en grupos que incluyen alumnado 0-6 (García-González y Schenetti, 2019) han resultado en experiencias didácticas relevantes.

Aquí se presenta una secuencia didáctica para este ciclo desde la perspectiva de la vida en el medio rural, atribuyéndole la importancia que merece, y facilitando nuevas experiencias con las abejas, de forma que el alumnado vea las abejas y la apicultura como parte integrante de su propio entorno cercano.

Los objetivos de esta intervención educativa entroncan en el objetivo para este primer ciclo de educación infantil del área de conocimiento del entorno de observar y explorar el entorno inmediato, centrado en los seres vivos (biodiversidad) a través de las abejas y sus relaciones. Dentro de esa área, en esta experiencia didáctica se trabaja especialmente en:

- Observar y explorar los objetos y materiales presentes en el medio que nos rodea.

- Respetar y cuidar los materiales y seres vivos del medio; acercarse y vincularse con la naturaleza a través de la observación de algunas características de los seres vivos, centrado en las abejas, su historia natural y productos.

- Iniciarse en el conocimiento del mundo físico a través de la observación, manipulación y experimentación relacionada con las abejas y apicultura.

- Disfrutar con actividades al aire libre y en contacto con la naturaleza a partir de la exploración del entorno inmediato.

\section{Contexto y metodología}

La intervención educativa se ha desarrollado en el curso 2018/2019 en la Escuela de Educación Infantil El Franco, de primer ciclo, situada en la zona rural del occidente de Asturias. Localizada en las afueras de la capital del municipio, está inserta en un entorno rural con amplias zonas verdes y mosaico de prados, cultivos y bosquetes en las cercanías. Dispone de patio escolar con zona con vegetación y acceso al huerto escolar. El alumnado reside en el ámbito rural cercano, mayoritariamente de familias trabajadoras y muy homogéneo culturalmente. Se han enfocado las actividades teniendo en cuenta el nivel de desarrollo y posibilidades concretas del grupo de 2-3 años (18 estudiantes), puesto que la primera autora era su tutora desde su entrada en la escuela, conociendo así sus peculiaridades y características personales.

Este primer ciclo está regulado en Asturias en el Decreto 13/2014, de 3 de diciembre, que marca que las actuaciones educativas se deberían basar en experiencias, sensaciones, actividades y juegos, y que deberían orientarse a generar satisfacción de experimentar, conocer, explorar, aprender, descubrir, investigar... planteamientos y metodologías muy adecuadas para los procesos de enseñanza-aprendizaje de las ciencias, y en los que se enmarca esta intervención educativa. Por lo tanto, se ha diseñado una secuencia de actividades a desarrollar en asamblea, y actividades cooperativas, en las que el resultado se alcanza conjuntamente con la participación de todo el alumnado, planteadas de manera que resulten atractivas y motivadoras, aportando el componente lúdico para favorecer un mejor aprendizaje. En ellas, la función de la educadora será la de guiar y acompañar en la elaboración de los diferentes aprendizajes, reforzando los logros, ayudándoles para que superen sus dudas, miedos o dificultades, y la del alumnado descubrir y aprender, ofreciéndoles la oportunidad de equivocarse y volver a intentarlo, perfeccionando así sus conocimientos y aprendizajes. Se ha procurado trasladar también el aprendizaje fuera del aula, incorporando actividades en el huerto escolar, en el patio escolar y en un prado 
cercano, que tuvieran como eje ese acercamiento en el entorno rural al medio natural a través de la apicultura. A este respecto, resulta oportuno indicar que la primera autora dispone de un pequeño colmenar, de donde han provenido las abejas, colmena y material de apicultura.

\section{Desarrollo de la innovación educativa}

La experiencia didáctica se ha implementado en tres semanas consecutivas. Las actividades se realizaron una al día, sobre las 10.30 horas, después del aperitivo y tras el cepillado de dientes. La duración máxima fue de 45 minutos, debido a la edad del alumnado, atendiendo su ritmo de atención para definir los momentos de corte.

La experiencia didáctica ha combinado asambleas (introductoria sobre lo que conocían de las abejas y su historia natural, incluyendo morfología y función de las castas y el ciclo de vida; otra específica sobre los problemas que tienen las abejas con especies invasoras y contaminación) y lecturas sobre las abejas, actividades manipulativas en el aula (observación de abejas, herramientas y productos apícolas, sobre cómo pican las abejas), experiencias en el huerto escolar (plantar flores para las abejas, colocar trampas para especies invasoras, observar insectos), actividades plásticas (simulación de panal y abejas) y una salida de campo a un prado cercano. Para las actividades manipulativas y de experiencias en el aula y el exterior se ha requerido la creación de diversos recursos didácticos, como fotografías, vídeos y abejas y otros artrópodos encapsulados (ver material anexo). El esquema de actividades y su cronograma puede consultarse en la Figura 1.

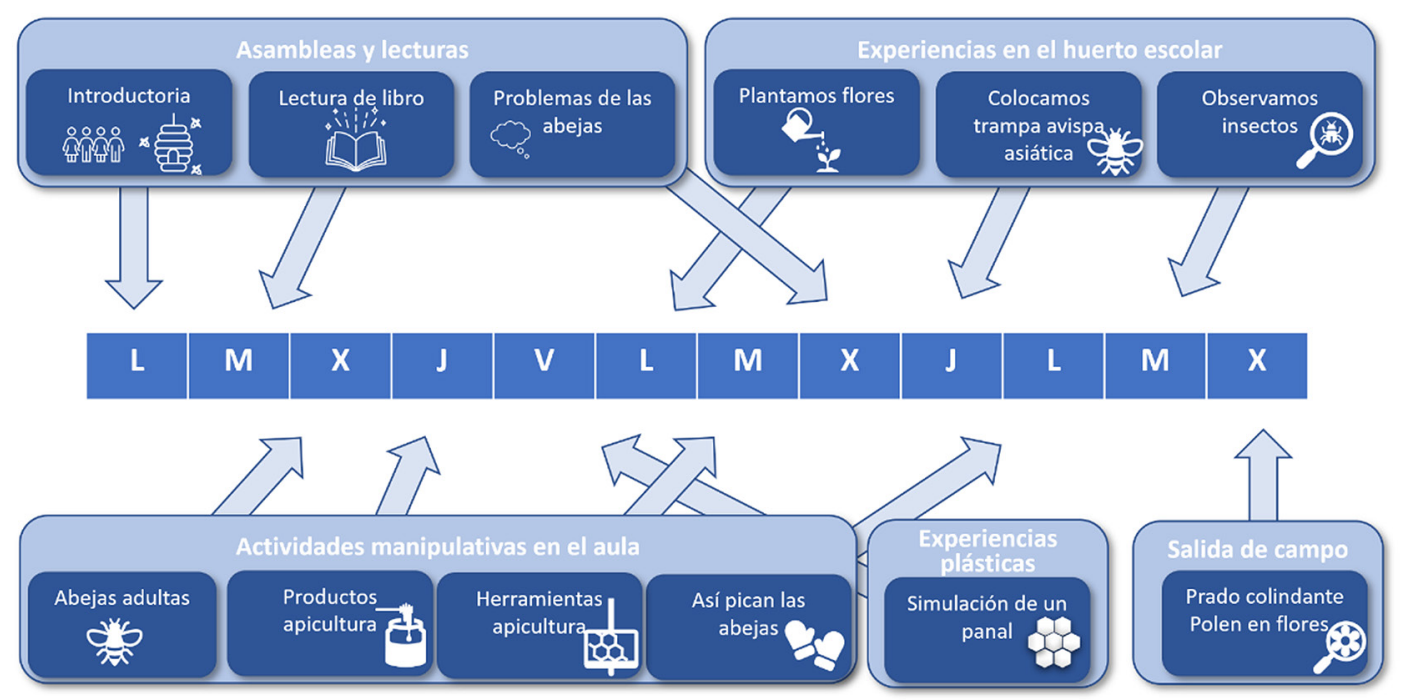

Figura 1. Esquema y cronograma de la experiencia didáctica (elaboración propia)

Se ha prestado atención al lugar donde se produce el aprendizaje, partiendo de aquel al que estaba más acostumbrado el alumnado (el aula), y organizando actividades educativas en el patio y el huerto escolar, para finalmente salir de la escuela a un prado cercano. En la Tabla 1 se muestra el lugar de aprendizaje de cada actividad, así como los objetivos específicos que se buscaban con cada una. La descripción más pormenorizada de cada actividad se proporciona en el Anexo 1. 
Tabla 1. Lugar de aprendizaje, temporalización ( $T$, min) y objetivos específicos de las actividades. Tipo actividad: $\mathrm{A}=$ asamblea; $\mathrm{H}=$ experiencias huerto escolar; $\mathrm{M}=$ manipulativa; $\mathrm{P}=$ plástica; $\mathrm{S}=$ salida.

\begin{tabular}{|c|c|c|c|c|c|}
\hline & Actividad & Tipo & Lugar & $\mathbf{T}$ & Objetivos específicos \\
\hline 1 & Inicial abejas & $A$ & Aula & $20-30$ & $\begin{array}{l}\text { Interesarse por la vida de las abejas. } \\
\text { Familiarizarse con las abejas. }\end{array}$ \\
\hline 2 & Lectura libro & $A$ & Aula & 20 & $\begin{array}{l}\text { Disfrutar con la escucha de cuentos. } \\
\text { Adentrarse en la vida de las abejas. }\end{array}$ \\
\hline 3 & $\begin{array}{l}\text { Observación } \\
\text { abejas }\end{array}$ & $M$ & Aula & $20-30$ & $\begin{array}{l}\text { Explorar características y texturas abejas. } \\
\text { Utilizar habilidades motrices. } \\
\text { Vivenciar la sensación que produce el contacto } \\
\text { directo con estos insectos. } \\
\text { Desarrollar capacidades sensoriales para } \\
\text { favorecer curiosidad por el entorno inmediato. }\end{array}$ \\
\hline 4 & $\begin{array}{l}\text { Productos } \\
\text { apícolas }\end{array}$ & M & Aula & 20 & $\begin{array}{l}\text { Degustar productos típicos abejas. } \\
\text { Utilizar habilidades sensoriales: gusto, olfato, } \\
\text { tacto y vista. } \\
\text { Experimentar con distintas materias primas que } \\
\text { ofrecen estos insectos. }\end{array}$ \\
\hline 5 & $\begin{array}{l}\text { Simulación } \\
\text { panal }\end{array}$ & $P$ & Aula & 20 & $\begin{array}{l}\text { Experimentar con diferentes formas de expresión } \\
\text { plástica. } \\
\text { Disfrutar con utilización de distintas técnicas y } \\
\text { recursos básicos de lenguaje plástico. } \\
\text { Descubrir y utilizar diferentes formas de } \\
\text { comunicación. }\end{array}$ \\
\hline 6 & $\begin{array}{l}\text { Plantamos } \\
\text { flores }\end{array}$ & $\mathrm{H}$ & Huerto & 45 & $\begin{array}{l}\text { Disfrutar del contacto con la naturaleza y con el } \\
\text { entorno. } \\
\text { Proyectar nuevas vivencias a través de la rutina } \\
\text { de la escuela. } \\
\text { Descubrir y experimentar con diferentes formas } \\
\text { de trabajar en la tierra. }\end{array}$ \\
\hline 7 & $\begin{array}{l}\text { Herramientas } \\
\text { apicultura }\end{array}$ & $M$ & Aula & $20-30$ & $\begin{array}{l}\text { Manipular distintos utensilios apicultura. } \\
\text { Familiarizarse con estos materiales. }\end{array}$ \\
\hline 8 & $\begin{array}{l}\text { Problemas } \\
\text { abejas }\end{array}$ & $A$ & Aula & 20 & $\begin{array}{l}\text { Visualizar ácaros varroa y avispón asiático } \\
\text { Mostrar interés hacia los seres vivos. }\end{array}$ \\
\hline 9 & $\begin{array}{l}\text { Trampa avispa } \\
\text { asiática }\end{array}$ & $\mathrm{H}$ & Huerto & $20+20$ & $\begin{array}{l}\text { Acercamiento y vinculación con la naturaleza. } \\
\text { Disfrutar del contacto con la naturaleza y con el } \\
\text { entorno. } \\
\text { Proyectar nuevas vivencias a través de la rutina } \\
\text { de la escuela. }\end{array}$ \\
\hline 10 & Así pican & M & $\begin{array}{l}\text { Patio, } \\
\text { Aula }\end{array}$ & 20 & $\begin{array}{l}\text { Adquirir nuevos conocimientos sobre abejas. } \\
\text { Mostrar actitud serena y participativa en las } \\
\text { situaciones relacionadas con el cuidado. } \\
\text { Prevenir biofobia. }\end{array}$ \\
\hline 11 & $\begin{array}{l}\text { Observamos } \\
\text { insectos }\end{array}$ & $\mathrm{M}, \mathrm{H}$ & Huerto & 20 & $\begin{array}{l}\text { Acercarse y vincularse con la naturaleza. } \\
\text { Observar características seres vivos. }\end{array}$ \\
\hline 12 & Salida campo & $S$ & Prado & 45 & $\begin{array}{l}\text { Mostrar interés por el medio en el que viven. } \\
\text { Disfrutar del pleno contacto con la naturaleza. }\end{array}$ \\
\hline
\end{tabular}




\section{Resultados y consideraciones finales}

Tanto el tema elegido, las abejas, como la realización de las mismas al aire libre y en contacto con la naturaleza han sido muy bien acogidas entre el alumnado. Las más exitosas han sido las actividades manipulativas en el aula, las experiencias en el huerto escolar y manipulativas, en las que cada estudiante ha podido vivir el contacto con la abeja, con los productos de la colmena, así como utilizar el ahumador, preparación y colocación de la trampa, la plantación en el huerto..., resultando muy novedoso y llamativo.

Es destacable que el alumnado pedía alargar las experiencias manipulativas y sensoriales en todas las actividades seguir disfrutando con ellas. Las asambleas, lectura de libros y vídeos, también han tenido buena acogida, si bien en la medida que podían manipular los materiales. En las actividades en el interior del aula el alumnado se empezaba a cansar antes, y se procedía a una desconexión gradual con la actividad (continuaban un poco más quienes seguían interesados en ella, mientras que se proporcionaba actividades alternativas al resto). Las propuestas plásticas han sido satisfactorias puesto que el alumnado ha disfrutado con su realización y han servido de recordatorio de lo trabajado los otros días.

Esta experiencia educativa ha profundizado en la importancia de la educación en contacto con el medio que nos rodea, el respeto hacia la naturaleza y las actividades del mundo rural, a través del empleo de las abejas. Además, se ha mostrado que en esta etapa es posible introducir propuestas de actividades novedosas, manipulativas y en contacto con la naturaleza, tal y como se han desarrollado en esta experiencia, que capten la atención del alumnado. En demasiadas ocasiones, en el primer ciclo de Educación Infantil, el contacto con la naturaleza está limitado a la hora de los recreos, que en muchos casos no se desarrollan en patios con vegetación, y no está incluido en las programaciones didácticas como actividades a realizar al aire libre. Esta secuencia didáctica sobre las abejas muestra las posibilidades de fomentar desde las escuelas e incluir más actividades de este tipo, con empleo del patio y el huerto escolar, que son algo más comunes en el segundo ciclo de la etapa (Aragón, Sánchez y Enríquez, 2020; Cantó, de Pro y Solbes, 2016), así como las experiencias sensoriales para un acercamiento científico al entorno natural (Mateo, Ferrer, Mazas, y Cascarosa, 2020; Puig, López-Lozano y García, 2020), que puedan generar emociones positivas hacia el medio natural (Lumber et al., 2017). De hecho, la inclusión de alguna salida didáctica adicional por los alrededores, como comienzo de la experiencia o como su extensión, podría incrementar estos aspectos positivos. El alumnado ha disfrutado y ha aprendido, atendiendo a las observaciones realizadas durante el curso. A su vez, se le ha dado la posibilidad de explorar, manipular, experimentar..., todo ello en un clima de seguridad; se han equivocado y han corregido ellos mismos volviendo a intentarlo (ver las indicaciones sobre reconocimiento de las abejas y cómo localizar polen en las flores en el Anexo 1). Y eso, conectando, además de con la naturaleza, con actividades propias de un entorno rural, como es el caso de la apicultura, en un momento en el que también existe una cierta desconexión entre los niños de entornos rurales y ese mismo entorno rural. 


\section{Referencias bibliográficas}

Almeida, A., García Fernández, B. y Strecht-Ribeiro, B. (2020) Children's knowledge and contact with native fauna: a comparative study between Portugal and Spain. Journal of Biological Education, 54(1), 17-32, DOI: https://dx.doi.org/10.1080/00219266.20 18.1538017

Aragón, L., Sánchez, S. y Enríquez, J. M. (2020). El discurso científico en la etapa de infantil en el contexto del huerto ecológico escolar. Revista Eureka sobre Enseñanza y Divulgación de las Ciencias, 18(1), 1103. DOI: https://doi.org/10.25267/Rev_ Eureka_ensen_divulg_cienc.2021.v18.i1.1103

Ardoin, N. M., y Bowers, A. W. (2020). Early childhood environmental education: A systematic review of the research literature. Educational Research Review, 31, 100353. DOI: https://doi.org/10.1016/j.edurev.2020.100353

Atkinson, K. (2018). Wasps-Bees-Mushrooms-Children: reimagining multispecies relations in Early Childhood Pedagogies. Canadian Children, 40(2), 67-79. DOI: https://doi. org/10.18357/jcs.v40i2.15180

Bartolomé, R. y del Pozo, M. (2009). Didáctica de la educación infantil. Madrid: McGrawHill.

Bejcek, J. R., Curtis-Robles, R., Riley, M., Brundage, A., Hamer, S. A. y Hamer, G. L. (2018). Clear Resin Casting of Arthropods of Medical Importance for Use in Educational and Outreach Activities. Journal of Insect Science, 18(2), 34: 1-4. DOI: https://dx.doi. org/10.1093/jisesa/iey030

Cantó Doménech, J., de Pro Bueno, A. y Solbes, J. (2016). ¿Qué ciencias se enseñan y cómo se hace en las aulas de educación infantil? La visión de los maestros en formación inicial. Enseñanza de las ciencias, 34(3), 25-50. DOI: http://dx.doi.org/10.5565/rev/ ensciencias.1870

Colmenar Orzales, C. (1989). La formación de maestras en el método educativo de Fröbel en España. Revista de Educación, 290: 135-158.

Corraliza, J. A. y Collado, S. (2011). La naturaleza cercana como moderadora del estrés infantil. Psicothema, 23(2), 221-226.

Cutter-Mackenzie, A., Edwards, S., Moore, D. \& Boyd, W. (2014) Young Children's Play and Environmental Education in Early Childhood Education. SpringerBriefs in Education. Springer, Cham.

Daynes, K. y Pym, C. (2017) ¿Quiénes son las abejas? Londres: Usborne.

Echols, J. C. (1999). Buzzing a hive: teacher's guide. Berkeley: GEMS, University of California.

Fisher, M.L., Miller, D.M. y Jamison, K. (2014). The buzz about bees: Honey bee biology and behavior. Blacksburg: Virginia State University.

Freire, H. (2011). Educar en verde: Ideas para acercar a niños y niñas a la naturaleza. Barcelona: Grao.

García, E., Rosado, M. y Hernaz, J. (2012). La colmena viajera. Guía para el docente. Gijón: Asociación Española de Entomología, Jardín Botánico Atlántico y Centro Iberoamericano de Biodiversidad.

García-González, E. y Schenetti, M. (2019) Las escuelas al aire libre como contexto para el aprendizaje de las ciencias en infantil. El caso de la Scuola nel BoscoVilla Ghigi. Revista Eureka sobre Enseñanza y Divulgación de las Ciencias, 16(2), 2204. DOI: https://doi.org/10.25267/Rev_Eureka_ensen_divulg_cienc.2019.v16.i2.2204 
Hart, S. (1987). The Honey bee across the curriculum. Southern African Journal of Environmental Education, 4, 15-18.

Inoue, M. (2018). Fostering an ecological worldview in children: rethinking children and nature in early childhood education from a Japanese perspective. En: Cutter-Mackenzie A., Malone K., Barratt Hacking E. (eds) Research Handbook on Childhoodnature. Springer International Handbooks of Education. Springer, Cham. DOI: https://doi.org/10.1007/978-3-319-51949-4_55-1

Ives, C. D., Abson, D. J., von Wehrden, H., Dorninger, C., Klaniecki, K., y Fischer, J. (2018). Reconnecting with nature for sustainability. Sustainability science, 13(5), 13891397. DOI: https://doi.org/10.1007/s11625-018-0542-9

Kesebir, S. y Kesebir, P. (2017). A growing disconnection from nature is evident in cultural products. Perspectives on Psychological Science, 12(2), 258-269. DOI: https://doi. org/10.1177/1745691616662473

Lemelin, R. H. y Yen, A. (2015) Human-spider entanglements: understanding and managing the Good, the Bad, and the Venomous. Anthrozoös, 28:2,215-228. DOI: http:// dx.doi.org/10.1080/08927936.2015.11435398

Lemelin, R. H., Harper, R. W., Dampier, J., Bowles, R. y Balika, D. (2016) Humans, insects and their interaction: a multi-faceted analysis. Animal Studies Journal, 5(1), 65-79.

Louv, R. (2008) Last child in the woods: Saving our children from nature-deficit disorder. Chapel Hill: Algonquin books.

Louv, R. (2016). Vitamin N: The essential guide to a nature-rich life. Londres: Algonquin Books.

Lumber, R., Richardson, M. y Sheffield, D. (2017). Beyond knowing nature: Contact, emotion, compassion, meaning, and beauty are pathways to nature connection. PloS ONE, 12(5), e0177186. DOI: https://doi.org/10.1371/journal.pone.0177186

Mateo González, E., Ferrer Bueno, L.M., Mazas Gil, B. y Cascarosa Salillas, E. (2020). ¿Entras a la cueva? Una experiencia multisensorial para trabajar las Ciencias en la etapa de Educación Infantil. Ápice. Revista de Educación Científica, 4 (2), 51-62. DOI: https:// doi.org/10.17979/arec.2020.4.2.5755

Montero. B., Conde, M.C. y Sánchez, J.S. (2016). Investigamos las abejas a través de una propuesta en el aula de educación infantil. En J. L. Bravo Galán (ed.), 27 Encuentros de Didáctica de las Ciencias Experimentales (pp. 1149-1157). Badajoz: Universidad de Extremadura.

Nxumalo, F. (2018). Stories for living on a damaged planet: environmental education in a preschool classroom. Journal of Early Childhood Research, 16(2), 148-159. DOI: https://doi.org/10.1177/1476718X17715499

Puig Gutiérrez, M., López-Lozano, L. y García Rodríguez, R. (2020) Experimentando con los sentidos: un rincón de ciencias en Educación Infantil. Didáctica de las Ciencias Experimentales y Sociales, 39, 117-134. DOI: https://doi.org/10.7203/ DCES.39.16893

Pyle, R. M. (2003). Nature matrix: reconnecting people and nature. Oryx, 37(2), 206-214. DOI: https://doi.org/10.1017/S0030605303000383

Rodríguez-Loinaz, G., Toral, N. y Palacios-Agundez, I. (2018). Bee-Plant Relationship in Early Childhood: a study through the analysis of children's drawings. Proceedings, 2(21), 1351. DOI: https://doi.org/10.3390/proceedings2211351 
Sensat, R. (1933). Los estudios de la naturaleza en la escuela primaria. Revista de Pedagogía, 141: 391-396.

Torres-Porras, J., Alcántara, J., Arrebola, J.C., Rubio, S.J. y Mora, M. (2017). Trabajando el acercamiento a la naturaleza de los niños y niñas en el Grado de Educación Infantil. Crucial en la sociedad actual. Revista Eureka sobre Enseñanza y Divulgación de las Ciencias, 14 (1), 258-270. Recuperado de: http://hdl.handle.net/10498/18860

Valin, A., Moledo, L., López Maceiras, M. y García-Rodeja, I. (2012). Un proyecto de ciencias en el aula de infantil: las abejas. En J. M. Domínguez Castiñeiras (ed.), XXV Encuentro de Didáctica de las Ciencias Experimentales (pp. 787-794). Santiago de Compostela: Universidade de Santiago de Compostela \& APICE.

Vega, S. (2006). Ciencia 0-3: laboratorios de ciencias en la escuela infantil. Barcelona: Graó. 


\section{Anexo 1: Descripción de las actividades}

La ordenación temporal de las actividades en la experiencia didáctica puede consultase en la Figura 1, mientras que su organización espacial y objetivos específicos sen encuentran en la Tabla 1. La duración temporal de la Tabla 1 fue la máxima para cada actividad, pero no todo el alumnado continuó durante todo ese tiempo. Atendiendo a la flexibilidad propia de la etapa, según el interés mostrado en la actividad, el alumnado que quería continuarla siguió con ella más tiempo que el que iba perdiendo el interés, al que se ofrecía pasar a otras actividades. Se expone a continuación la descripción de dichas actividades, organizadas en cuanto al tipo de actividad (asamblea y lecturas, manipulativas en el aula, experiencias en el huerto escolar, salida de campo y actividades plásticas).

\section{Actividades de asamblea y lecturas}

\section{Asamblea inicial sobre la abeja y su historia natural}

La finalidad de la actividad es realizar una evaluación diagnóstica de los conocimientos y experiencias del alumnado, para saber desde donde se parte, además de comenzar a hablar sobre las abejas. Se llevó al aula una colmena (vacía), un panal con miel y abejas obreras adultas vivas en una caja de observación. Colocados en asamblea, se les mostró lo traído y se comenzó realizando preguntas diagnósticas que dirigieran la asamblea hacia el tema que se buscaba tratar en este aprendizaje ¿Sabéis que insecto es éste?, ¿Dónde viven?, ¿Qué nos aportan?, ¿A quién le gusta la miel?... La relación de la abeja con la miel quedaba clara rápidamente, la identificación inicial de los insectos reales como abejas costó algo más. Se les proyectó las fotografías y vídeo creados sobre las distintas fases y castas de las abejas en la colmena.

Durante la asamblea se distribuyó el aperitivo, de forma que también se siguió hablando del tema mientras el alumnado lo comía (con la ayuda de la maestra, de acuerdo con su nivel madurativo).

\section{Lectura del libro ¿Quiénes son las abejas? (Daynes y Pum, 2017)}

Con la lectura de este libro y su manipulación ante el alumnado para que visualizara las imágenes, se pretendía que les sirviera como referente para conocer un poco más sobre las abejas, ya que posee unas ilustraciones adaptadas a su edad y también cuenta con un sistema de solapas que desvelan preguntas gráficamente. Sin ser dibujos morfológicamente precisos, sí que las abejas presentaban una coloración adecuada, el cuerpo mostrando las partes principales (cabeza, tórax, abdomen), con cuatro alas y seis patas saliendo del tórax, lo que representa una aproximación aceptable al concepto de abeja, sirviendo de puente entre sus ideas de lo que es una abeja y los insectos reales.

\section{Problemas de las abejas}

Se realiza una asamblea con manipulación de invertebrados posterior para contribuir a que el alumnado conozca las principales amenazas de la abeja. Se incidió en la distinta relación con ambas especies, donde viven, las fases de las que se alimentan y problemas que presentan. Para facilitar el acercamiento a esas cuestiones, teniendo en cuenta la edad del alumnado, se les mostró la varroa y la avispa asiática, diciéndoles que el ácaro vive en la colmena con las abejas y se come sus larvas y las alas antes de nacer. También, que la avispa caza las abejas en pleno vuelo y se las lleva para su nido para alimentar a sus crías. Además, se trató el problema ambiental del uso masivo de pesticidas, mediante una versión simplificada en la que se indicaba que el problema venía porque las abejas se alimentan del polen y del néctar que obtienen de las flores y plantas que han sido sulfatadas al emplear pesticidas en la agricultura.

\section{Actividades manipulativas en el aula}

\section{Observación de abejas adultas}

Se observaron/manipularon ejemplares adultos de las distintas castas de abejas (reina, zángano y obrera). De ellos, únicamente los zánganos pueden ser manipulados vivos sin riesgo por el alum- 
nado, ya que carecen de aguijón, por lo que se decidió encapsular abejas muertas como recurso didáctico para facilitar manipulación y evitar estrés innecesario en los animales (ver material anexo). Al verlas el alumnado no las identificaban como abejas (las referían como "moscas"), pero tras indicarles que eran abejas, posteriormente sí que hacían referencia a ellas abejas. La actividad se realizó sobre la mesa del aula, proporcionándoles ejemplares encapsulados y alguno no encapsulado para sentir su tacto, verbalizando las diferencias al tiempo que los manipulaban. En este sentido, hay que tener en cuenta que el currículo del ciclo plantea como contenidos la observación de algunas características de los seres vivos, y la relación, aunque no sea directamente aplicable, entre funciones de cada casta y el desarrollo de profesiones humanas (sobre las que existen numerosas canciones infantiles), puede resultar de utilidad en las explicaciones de las funciones. Hubo una pequeña parte del alumnado que mostró ciertos reparos a los insectos no encapsulados, y que no los tocaron, pero sí que pudieron observar como el resto interactuaba con ellos, aprovechando así la actividad.

\section{Productos de la colmena}

La finalidad de la actividad fue mostrar que las abejas producen distintos recursos aprovechables por los seres humanos (miel, propóleo, cera, jalea real, polen y néctar), que el alumnado pudiera probarlos, manipularlos y tener experiencias multisensoriales (exploraciones con la vista, el tacto, el olfato y el gusto). La maestra también los probó y experimentó con las distintas texturas. Se comentó la forma de producción por parte de las abejas y su utilidad (para la abeja y para los seres humanos). La identificación de la miel como producto de las abejas fue muy rápida (si bien no el origen o el proceso), pero desconocían el resto.

\section{Materiales de apicultura}

Se pretendió que se familiarizaran con los utensilios (ahumador, pinzas de extracción, jaulas, marcador de reinas, guantes, traje, colmena vacía y panales) empleados en apicultura, que previamente les eran desconocidos, y con su uso. La maestra verbalizó qué era cada herramienta y su uso, a la vez que ellos las manipulaban y experimentaban con ellas. El traje para apicultura, con su careta de rejilla, y el ahumador fueron los que más llamaron su atención.

\section{Así pican las abejas - taller de enfermería}

Que las abejas pueden picar es algo que tenía claro el alumnado, aunque no haya tenido una experiencia directa de ese tipo. Cómo lo hacen, y qué implicaciones tiene para la abeja y para la persona no es algo que tengan tan claro. Teniendo en cuenta la posible ambigüedad de sentimientos que pueden generar entre el alumnado las abejas y otros artrópodos que pueden picar (Lemelin y Yen, 2015; Lemelin, Harper, Dampier, Bowles y Balika, 2016), se optó por incluir una actividad en la que se expusieran estas cuestiones y se pudieran reconducir sentimientos negativos o de rechazo. La maestra, llevando guantes de apicultor, cogió una abeja obrera y esta clavó su aguijón en el guante como mecanismo de defensa. El alumnado observó que, con la picadura, el aguijón quedaba enganchado en el tejido y que con él se desprendía la parte interna del abdomen (se les explicó que seguía inyectando el veneno de esa forma) y que la abeja moría. Se comentó que en algunos lugares se comercializa este producto (el veneno de abeja), pero que puede resultar muy peligrosa la picadura de abejas y avispas si se es alérgico, y que no debemos molestar a las abejas, ya que si no se sienten amenazadas no nos picarán. A continuación, se retiró el aguijón con una pinza y puso una tirita al guante, donde picó la abeja.

\section{Experiencias en el huerto escolar}

\section{Plantamos flores en nuestro huerto}

Con el fin de atraer más insectos polinizadores, entre ellos las abejas, se trasplantaron plantas de flor ornamental (petunias, alegrías, surfinias) en el huerto escolar, que hasta ese momento tenía solo hortalizas. En el huerto se facilitó al alumnado azadas y palas, y con nuestra ayuda colocaron las plantas separadas (Figura 2). Disfrutar con actividades al aire libre y en contacto con la naturaleza, salir de la rutina de la escuela y descubrir y experimentar con las diferentes formas de trabajar 
en la tierra fueron objetivos conseguidos en esta actividad. Durante las actividades al aire libre se aprovechó para trasladar realizar las actividades del recreo a continuación, de forma que el alumnado pudiera moverse libremente entre las relativas al proyecto y las propias del recreo según sus inquietudes y necesidades.

\section{Construimos trampa para la avispa asiática y distinguimos insectos}

Entre las principales amenazas actuales en Asturias para las abejas y las explotaciones apícolas está la expansión de la especie invasora avispa asiática. Tras plantear y comentar la situación en el aula, se realizó una trampa sencilla con una botella de plástico agujereada, y se exploró el concepto de mezcla para la realización del atrayente con agua, levadura prensada y azúcar. Se colgó en la valla que limitaba el huerto y patio escolar (Figura 2).

La trampa permaneció cuatro días, se volvió a salir al huerto, la maestra la descolgó y se observaron los insectos caídos. Se debatió sobre cómo habían llegado hasta allí y por qué no habían salido. A continuación, se vació el contenido sobre una superficie lisa y se ordenó a los insectos por tamaño, viendo cual era cada uno. También se les dio la oportunidad de manipularlos y tocarlos, con los mismos condicionantes que los observados en la actividad con abejas adultas.
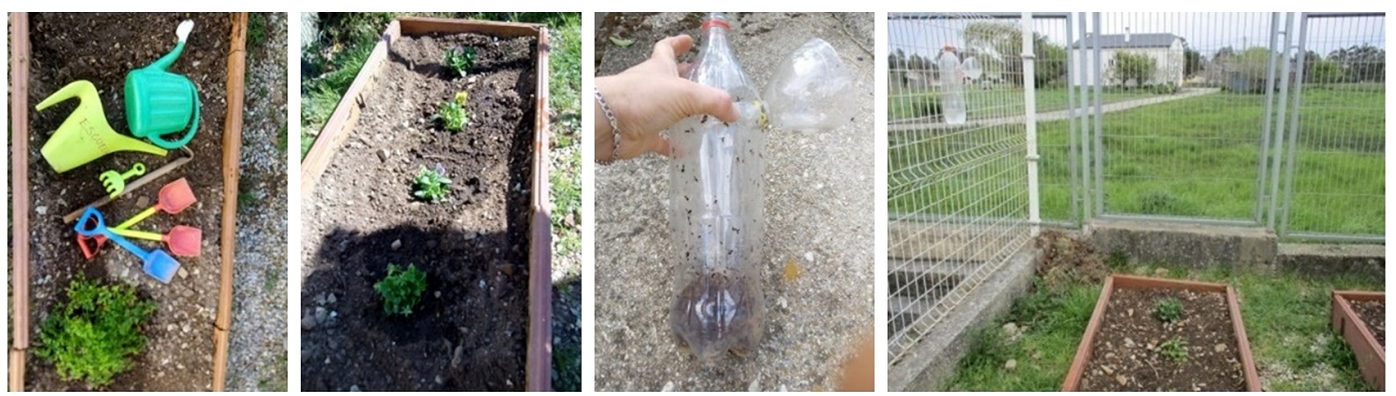

Figura 2. Experiencias en el huerto escolar: materiales para plantar las flores, plantas colocadas en el huerto, trampa para avispa asiática y disposición en el huerto escolar (elaboración propia)

\section{Salida de campo: encontramos polen en las flores}

Se buscó que el alumnado comprendiera dónde se encuentra el polen que recogen las abejas. Mostrar interés por el medio en el que viven y disfrutar del contacto con la naturaleza fueron objetivos alcanzados. Se salió más allá de la escuela, a un prado colindante. El alumnado debía buscar flores, y, tras recoger una, observar su interior. Dependiendo de la flor, color, cantidad de polen y su visibilidad resultó variable, siendo necesario que el alumnado se fijara y tocara estambres para que pudiera apreciar el polen. Su primera reacción para buscar el polen era coger las flores y olerlas, ya que en algunos casos era lo que hacían con sus familias con las flores, y el resto les fue imitando. Al indicarles que las tocaran en la parte central, en flores con abundante polen, al presionar los estambres sobre papel de cocina, dejó algunas manchas (igual que en nuestros dedos). Con ello aprendieron a localizar dónde se encontraba el polen y las diferencias de color entre el de las distintas flores (amarillo, blanco, rojo).

\section{Actividades plásticas: elaboración de un panal}

Se realizó una simulación de un panal, con los cartones centrales de rollos de papel higiénico cortados en aros estrechos y doblados de forma hexagonal para pegarlos juntos sobre una cartulina. Con unos tapones, limpiapipas y ojos se simularon abejas en algunas celdillas, de forma que les recuerde lo visto en vídeo y fotografías, comprobando diferencias. 


\section{Instrumentos y resultados de la evaluación}

La evaluación se llevó a cabo mediante la observación directa al alumnado durante la realización de las diferentes actividades, recogidas en el diario de aula y las listas de control, en donde se anotó la información recogida en la asamblea de conocimientos previos, así como en cada sesión. Se trata de una evaluación basada en la acción, procesual y destinada a poder producir cambios para lograr los aprendizajes deseados (Bartolomé y del Pozo, 2009). Las reflexiones realizadas sobre esas anotaciones se han dirigido mediante unos ítems previamente elegidos, que permitieran una adecuada retroalimentación para poder realizar los cambios oportunos (Tabla 2).

Tabla 2. Evaluación procesual realizada sobre el alumnado

\begin{tabular}{|c|c|c|}
\hline Indicadores & Valoración/Observación & Propuesta de mejora \\
\hline $\begin{array}{l}\text { Interacciona con todos } \\
\text { los elementos presentes } \\
\text { en el espacio, se mueve } \\
\text { libremente, experimenta } \\
\text { con ellos y genera opinión }\end{array}$ & $\begin{array}{l}\text { Mayoritariamente sí, 11\% } \\
\text { alumnado con interacción } \\
\text { limitada (timidez/miedo). }\end{array}$ & $\begin{array}{l}\text { Atención más personalizada, } \\
\text { se deja que parte del } \\
\text { alumnado realicen la actividad } \\
\text { previamente para que cojan } \\
\text { confianza con su desarrollo. }\end{array}$ \\
\hline $\begin{array}{l}\text { Disfruta con las experiencias } \\
\text { planteadas }\end{array}$ & $\begin{array}{l}\text { En unos casos realizando } \\
\text { la experiencia, en otros } \\
\text { observando. }\end{array}$ & $\begin{array}{l}\text { Aproximación diferente según } \\
\text { alumnado }\end{array}$ \\
\hline $\begin{array}{l}\text { Experimenta activamente } \\
\text { con los diferentes } \\
\text { materiales y recursos de la } \\
\text { naturaleza ofrecidos }\end{array}$ & $\begin{array}{l}\text { Aproximación diferente según } \\
\text { alumnado; mientras una parte } \\
\text { observaba sin participar, otra } \\
\text { quería alargar la actividad. }\end{array}$ & $\begin{array}{l}\text { Alargarla para quienes lo } \\
\text { piden, ofrecer otra actividad } \\
\text { a quienes se cansen de la } \\
\text { misma. }\end{array}$ \\
\hline $\begin{array}{l}\text { Interés por las actividades al } \\
\text { aire libre y en contacto con } \\
\text { la naturaleza }\end{array}$ & $\begin{array}{l}\text { Todo el alumnado; incluso } \\
\text { solicitaban seguir realizándolas. }\end{array}$ & $\begin{array}{l}\text { Incrementar experiencias en } \\
\text { el exterior }\end{array}$ \\
\hline $\begin{array}{l}\text { Interacciona de forma } \\
\text { equilibrada con el resto } \\
\text { del alumnado durante las } \\
\text { actividades, mostrando su } \\
\text { punto de vista, resaltando lo } \\
\text { positivo de las actividades } \\
\text { realizadas, siempre desde el } \\
\text { respeto y la cordialidad }\end{array}$ & $\begin{array}{l}\text { Sí, el alumnado que participaba } \\
\text { activamente; los que } \\
\text { permanecían viendo la actividad } \\
\text { no mostraron sensaciones } \\
\text { negativas, con excepción de las } \\
\text { interacciones con los insectos } \\
\text { no encapsulados, que en algún } \\
\text { caso mostraron algo de rechazo. }\end{array}$ & $\begin{array}{l}\text { Al interaccionar en gran grupo, } \\
\text { aquella parte del alumnado } \\
\text { que mostraba reparos a los } \\
\text { insectos, y que no los tocaron, } \\
\text { sí que pudieron observar } \\
\text { como el resto interactuaba } \\
\text { con ellos, aprovechando así la } \\
\text { actividad. }\end{array}$ \\
\hline $\begin{array}{l}\text { Reconoce la abeja frente a } \\
\text { otro insecto }\end{array}$ & $\begin{array}{l}\text { Al menos temporalmente sí, } \\
\text { frente a los mostrados }\end{array}$ & $\begin{array}{l}\text { Contrastar las ideas previas } \\
\text { con las abejas reales y otros } \\
\text { insectos. }\end{array}$ \\
\hline $\begin{array}{l}\text { Conoce las características } \\
\text { principales de la abeja: } \\
\text { insecto volador, elaborador } \\
\text { de la miel }\end{array}$ & Sí & $\begin{array}{l}\text { Dependiendo del interés } \\
\text { y posibilidades de cada } \\
\text { estudiante. }\end{array}$ \\
\hline $\begin{array}{l}\text { Disfruta de la realización de } \\
\text { actividades plásticas }\end{array}$ & En su gran mayoría, sí. & $\begin{array}{l}\text { Solo se presentó alguna } \\
\text { dificultad con la pintura de } \\
\text { dedos. }\end{array}$ \\
\hline $\begin{array}{l}\text { Muestra interés por la } \\
\text { escucha de cuentos }\end{array}$ & Sí, siempre & $\begin{array}{l}\text { Cada proyecto se apoya en } \\
\text { cuentos ilustrados, por lo que } \\
\text { tenían experiencia. }\end{array}$ \\
\hline
\end{tabular}


Tabla 2. Continuación. Evaluación procesual realizada sobre el alumnado

\begin{tabular}{|l|l|l|}
\hline Indicadores & Valoración/Observación & Propuesta de mejora \\
\hline $\begin{array}{l}\text { Exterioriza su interés por } \\
\text { realizar actividades de } \\
\text { asamblea e intercambio de } \\
\text { conocimientos }\end{array}$ & $\begin{array}{l}\text { Sí, de forma variable (ver } \\
\text { propuesta de mejora). }\end{array}$ & $\begin{array}{l}\text { En la asamblea se consiguió } \\
\text { la participación de todo el } \\
\text { alumnado y la comunicación, } \\
\text { no en todos los casos oral } \\
\text { (pero sí la escucha). Cuando } \\
\text { se detectaba que la atención } \\
\text { disminuía y se despistaban, se } \\
\text { cambiaba de actividad. }\end{array}$ \\
\hline
\end{tabular}

Para la autoevaluación realizada por la maestra de la experiencia didáctica, se definieron previamente una serie ítems que obligaran a la reflexión diaria sobre su desarrollo desde una actitud de autocrítica, que permitiera reconocer errores y aciertos en el tanto en cada actividad concreta como especialmente en el conjunto de la experiencia, buscando la mejora y reconduciendo la situación de ser necesario (Bartolomé y del Pozo, 2009). Desde la perspectiva de haber tutorizado a ese grupo desde su inicio en la escuela, se puede indicar que las actividades resultaron muy motivadoras, atractivas y novedosas, pudiendo llevarse a cabo con el alumnado, atendiendo a su edad y nivel de desarrollo. En todo momento se generó un clima de confianza y seguridad, tanto dentro como fuera del aula. Se consiguió respetar los distintos ritmos de aprendizaje, ayudando a cada estudiante individualmente cuando fue necesario, y se solventaron en la medida de lo posible los problemas surgidos.

Inicialmente se había planteado otra salida didáctica a un equipamiento educativo relacionado con el tema (un Aula de la Miel), pero no pudo realizarse por cuestiones operativas. En ese sentido, el desarrollo de la secuencia didáctica podría beneficiarse realizando una salida didáctica inicial al entorno inmediato, con el fin de ir ganando seguridad en mismo y poder generar más preguntas en el alumnado que le llevaran a desear aprender más sobre el tema. Incrementar las actuaciones manipulativas, y desplazar alguna de las actividades llevadas a cabo en el interior del aula al patio escolar (de permitirlo las condiciones meteorológicas, lo que no es siempre posible en la región), podría incrementar todavía más la motivación del alumnado, a tenor de los resultados obtenidos con ese tipo de actividades y lugar de aprendizaje en esta experiencia.

\section{Posibles extensiones de la experiencia}

Esta experiencia didáctica ha sido diseñada e implementada con éxito teniendo en cuenta las características concretas del primer ciclo de educación infantil en una escuela rural de pequeño tamaño. Sería posible extenderla en el tiempo, incluyendo una salida más y algunas actividades al aire libre, como se indica al comentar la evaluación de la experiencia, si la situación en el centro y con el alumnado es adecuada para ello. También la inclusión de alguna otra actividad plástica, de baile relacionado con la danza de las abejas o de observación, siempre atendiendo al nivel madurativo del alumnado.

Podrían implementarse estas actividades en el segundo ciclo de educación infantil, incrementado su complejidad según el curso e incorporando nuevas actividades y dramatizaciones (véase las recogidas en la bibliografía citada en la introducción), siempre según las posibilidades del centro educativo y grupo. 


\section{Material anexo: recursos didácticos generados}

Se han creado unos recursos específicos para la enseñanza aprendizaje sobre la historia natural de las abejas. Por una parte, se han realizado fotografías y vídeos de las distintas castas (reina, obrera, zángano) y fases (huevo, larva, pupa e imago) aprovechando una colmena modificada (Figura 3). Estas fotografías se han empleado proyectándolas durante las asambleas y pasándolas también impresas para ver las diferencias tras las explicaciones, así como ordenándolas en secuencias e identificándolas.

\section{Ciclo de vida de la abeja}

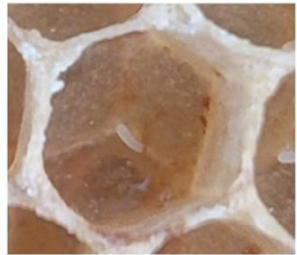

Huevo

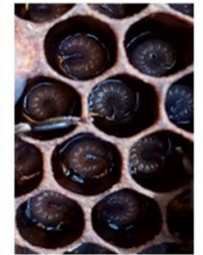

Larvas

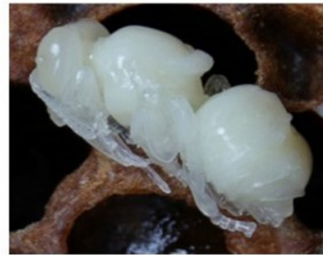

Pupa

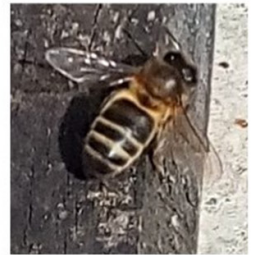

Adulto

\section{Las tres castas de la abeja}

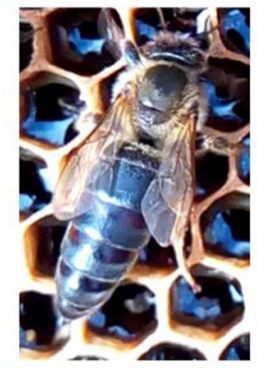

Reina

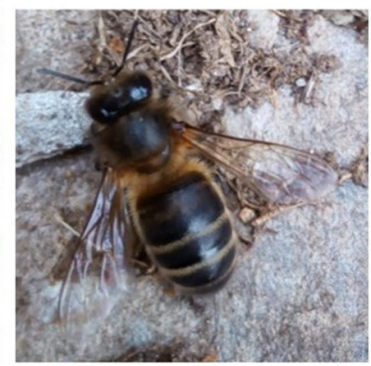

Obrera

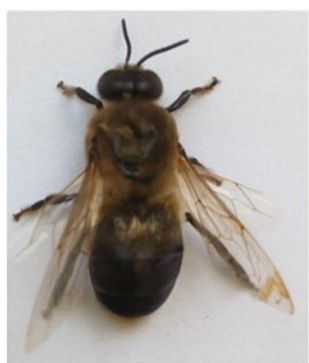

Zángano

\section{Distintas celdas en los panales}

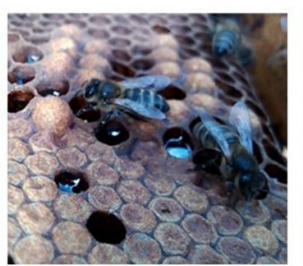

Zánganos (abultadas)

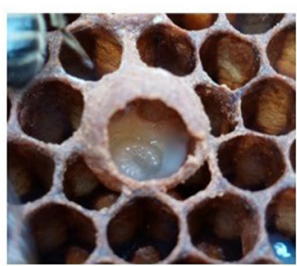

Día 5 (larva visible)

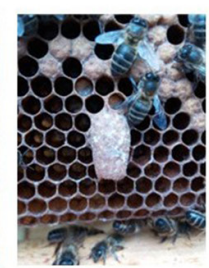

Realeras

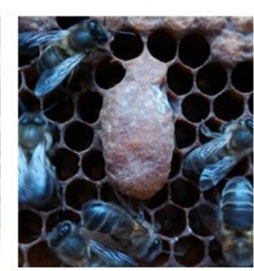

Día 10

Figura 3. Fotografías tomadas en la colmena modificada para servir de recursos didácticos sobre la historia natural de las abejas (elaboración propia)

Además, se han realizado ejemplares encapsulados de abejas adultas muertas. Las abejas encapsuladas no se deterioran ni pierden partes tras recibir las atenciones del alumnado, lo que facilita su reutilización en el tiempo. Así, se crea un recurso didáctico al que se puede recurrir con la frecuencia y tiempos que sean necesarios (o requeridos por el alumnado), sin necesidad de tener que recurrir al sacrificio de nuevos ejemplares. En ese sentido, el producto de la encapsulación de artrópodos realizada específicamente para educación se ha empleado con éxito en procesos de enseñanza-aprendizaje en distintos niveles educativos, siguiendo un planteamiento de arriba-abajo desde la Universidad, a la Educación Secundaria y la Educación Primaria (Bejcek et al., 2018), y existen versiones comerciales que pueden conllevar algunas implicaciones éticas, pero no se han detectado experiencias en la Educación Infantil, desarrolladas además desde este primer ciclo. 
Para la realización del encapsulado se empleó resina de poliéster transparente OCL y moldes cuadrados en los que verter y colocar los ejemplares. Se probó previamente con resina epoxi, pero los bloques resultantes resultaban poco transparentes, muy amarillentos, lo que falseaba los colores. Se vertió una primera capa de resina fundida, se colocó un individuo (muerto) sobre esa capa en cada molde, se recolocó en la forma elegida y se vertió el resto de la resina hasta llenar cada molde. El proceso de calentar la resina y verterla sobre el molde se realizó al aire libre.

Se encapsularon abejas adultas (obreras sobre todo, con y sin los característicos aglomerados de polen en los cestillos de sus patas traseras, zánganos y una reina) aprovechando para incluir también algunos ejemplares de la especie invasora avispón asiático o velutina y de del ácaro varroa, así como alguno de los que había apareció en la trampa (Figura 4).

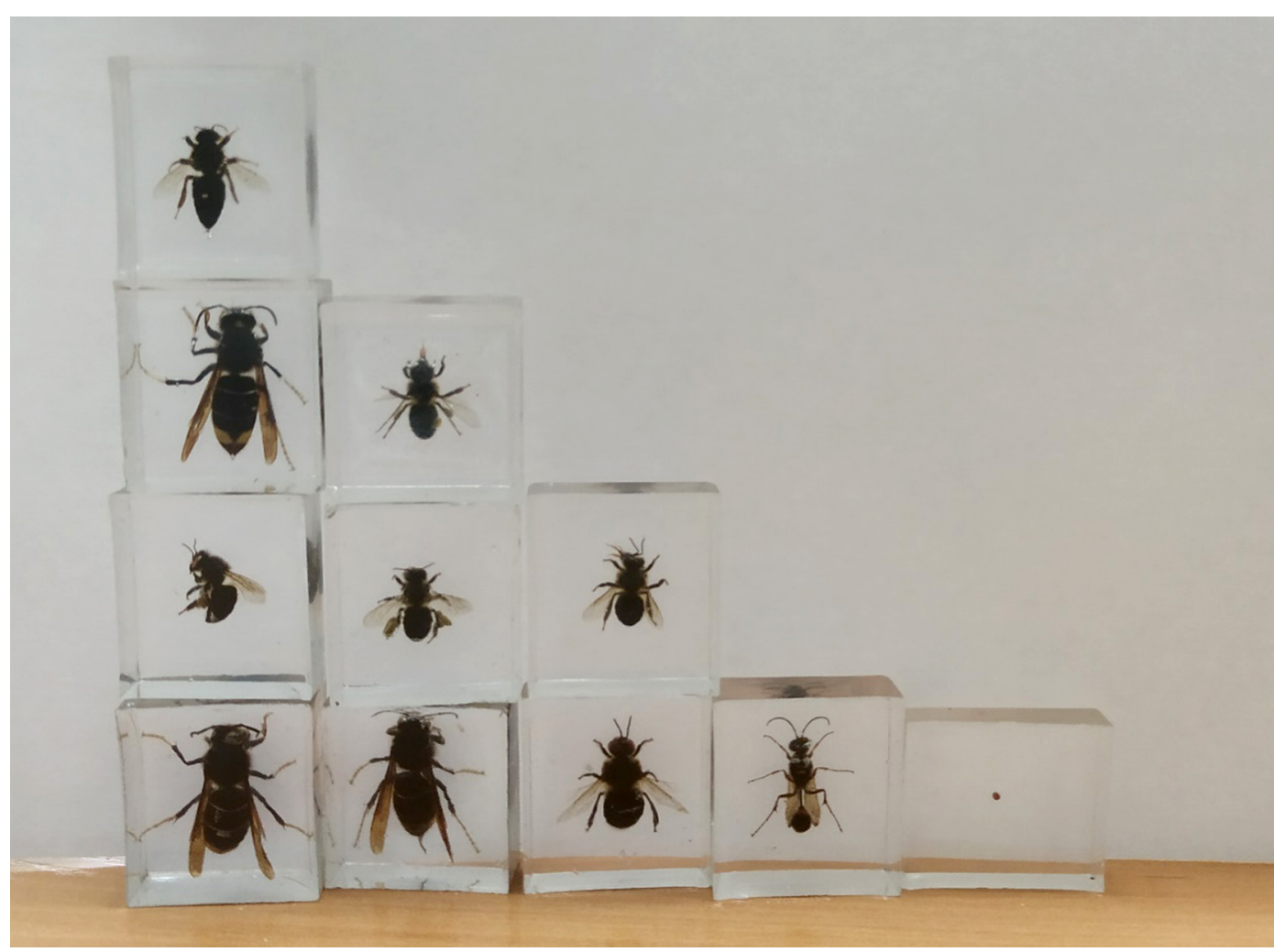

Figura 4. Aspecto final de los bloques de resina trasparente con los artrópodos incluidos (elaboración propia) 